\title{
A Glucosylceramide Synthase Inhibitor Protects Rats Against the Cytotoxic Effects of Shiga Toxin 2
}

\author{
CLAUDIA SILBERSTEIN, MARÍA S. LUCERO, ELSA ZOTTA, DIANE P. COPELAND, LI LINGYUN, HORACIO A. REPETTO, \\ AND CRISTINA IBARRA
}

Laboratorio de Fisiopatogenia, Departamento de Fisiología [C.S., M.S.L., E.Z., C.I.], Facultad de Medicina, Universidad de Buenos Aires, 1121 Buenos Aires, Argentina; Genzyme Corporation [D.P.C., L.L.], 211 Second Avenue, Waltham, Massachusetts 02451; Servicio de Pediatría [H.A.R.], Hospital Nacional Professor Alejandro Posadas, 1684 Buenos Aires, Argentina

\begin{abstract}
Postdiarrhea hemolytic uremic syndrome is the most common cause of acute renal failure in children in Argentina. Renal damage has been strongly associated with Shiga toxin (Stx), which binds to the globotriaosylceramide (Gb3) receptor on the plasma membrane of target cells. The purpose of the study was to evaluate the in vivo effects of C-9, a potent inhibitor of glucosylceramide synthase and Gb3 synthesis, on kidney and colon in an experimental model of hemolytic uremic syndrome in rats. Rats were i.p. injected with supernatant from recombinant Escherichia coli expressing Stx2 (sStx2). A group of these rats were orally treated with C-9 during $6 \mathrm{~d}$, from $2 \mathrm{~d}$ prior until $4 \mathrm{~d}$ after sStx 2 injection. The injection of sStx2 caused renal damage as well as a loss of goblet cells in colonic mucosa. Oral treatment with C-9 significantly decreased rat mortality to $50 \%$ and reduced the extension of renal and intestinal injuries in the surviving rats. The C-9 also decreased Gb3 and glucosylceramide expression levels in rat kidneys. It is particularly interesting that an improvement was seen when C-9 was administered $2 \mathrm{~d}$ before challenge, which makes it potentially useful for prophylaxis. (Pediatr Res 69: 390-394, 2011)
\end{abstract}

$\mathrm{P}$ ostdiarrhea hemolytic-uremic syndrome (HUS) is the most common cause of acute renal failure in children in Argentina and the second leading cause of chronic renal failure in children younger than $5 \mathrm{y}(1,2)$. The acute stage of the disease is marked by hypertension, edema, hematological abnormalities, and anuria $(1,3)$. Clinical and histological renal damages have been strongly associated with Shiga toxin type 1 and/or 2 (Stx1, Stx2) produced by Escherichia coli O157:H7 and other related bacterial strains frequently isolated from children with HUS, although strains expressing Stx2 are highly prevalent in Argentina (4). Shiga toxin (Stx) contains an A subunit monomer $(32 \mathrm{kD})$ bound noncovalently to five B subunits $(7.7 \mathrm{kD})(5)$. The B subunit pentamer binds to the glycolipid globotriaosylceramide (Gb3) on the plasma membrane of target cells. Then, Stx is internalized into the cell, and the A subunit exhibits RNA $N$ glycohydrolase activity and cleaves a specific adenine residue on the $28 \mathrm{~S}$ ribosomal RNA in the cytosol, thereby inhibiting protein synthesis. Therefore, Gb3 has been described to be the functional

Received May 5, 2010; accepted November 24, 2010.

Correspondence: Claudia Silberstein, Ph.D., Departamento de Fisiología, Universidad de Buenos Aires, Paraguay 2155 piso 6, 1121 Buenos Aires, Argentina; e-mail: csilber@fmed.uba.ar

Supported by grants from Universidad de Buenos Aires (CI: UBACYT M030 and CS UBACYT M440), the National Council of Research (CONICET, PIP 5587), and ANPCYT (PICT-0642). receptor for Stx found on the surface of target cells, producing Stx-mediated diseases $(6,7)$.

The kidney expresses relatively high levels of Gb3 compared with other organs, which may account, at least in part, for renal targeting in HUS. Gb3 is expressed in glomerular endothelial cells, podocytes, mesangial cells, and proximal tubule epithelial cells of the kidney $(5,8)$. The binding of Stxs to renal tubular epithelial cells expressing $\mathrm{Gb3}$ in vitro $(8,9)$ and in vivo $(10,11)$ has been shown to inhibit protein synthesis and induce apoptosis and necrosis. We have demonstrated that Stx2 holotoxin and its B subunit (Stx2B) inhibit water absorption across human renal tubular epithelial cell monolayers (12) and human colonic mucosa (13).

We have recently observed that C-9 (Genzyme, Waltham, MA), a specific inhibitor of glucosylceramide (GL1) synthase, decreases Gb3 expression levels and prevents the cytotoxic effects of Stx 2 on primary cultures of human renal tubular epithelial cells (HRTEC) (14). The rate-limiting first step in the biosynthesis of Gb3 and others glycosphingolipids is the reaction catalyzed by the enzyme GL1 synthase, which glycosylates the ceramide using uridine diphospho-glucose $(15,16)$. Different inhibitors of GL1 synthase have been identified and assayed for substrate inhibition therapy for treating several glycosphingolipidoses such as Fabry, Gaucher, Sandhoff, and Tay-Sachs disease $(17,18)$. These compounds can inhibit glycosphingolipid synthesis in cultured cells without inhibiting cell growth or raising intracellular ceramide levels (15). C-9, also referred to as Genz-123346, has been previously characterized as a potent and specific inhibitor of GL1 synthase that blocks the conversion of ceramide to GL1 (19). Previous studies showed that the C- 9 was well tolerated by mice and rats after multiple weeks of oral treatments (19). Other inhibitors of GL1 synthase that were highly effective to deplete Gb3 have been proposed for substrate inhibition therapy of Fabry disease (17). Additionally, Genz-112638, another potent GL1 synthase inhibitor has been assayed in a murine model of Gaucher disease to decrease the aberrant lysosomal accumulation of GL1 (18).

Abbreviations: Gb3, globotriaosylceramide; GL1, glucosylceramide; HUS, hemolytic uremic syndrome; LPS, lipopolysaccharide; MRM, multiple reaction monitoring; sCtrl, control supernatant; sStx2, supernatant from recombinant E. coli expressing Stx2; Stx, Shiga toxin; Stx2, Shiga toxin type 2 
In this study, we have assayed the in vivo effects of C-9, a potent inhibitor of GL1 synthase, in an experimental model of HUS in rats. Oral C-9 treatment significantly decreased rat mortality to $50 \%$. This treatment also decreased the Gb3 expression levels in kidneys and prevented the lesions of Stx2 in kidney and colon. The inhibition of Gb3 synthesis may be used as a potential treatment for protection against the pathological effects of Shiga toxin producing HUS. Preliminary reports of these experiments were presented in the VTEC2009, 7th International Symposium on Shiga toxin (Verocytotoxin)-producing Escherichia coli infection, Buenos Aires, Argentina, 10th to 13th May 2009.

\section{MATERIALS AND METHODS}

The C-9 [(1R, 2R)-nonanoic acid [2-(2', 3'-dihydro-benzo (1-4) dioxin6'-yl)-2-hydroxy-1-pyrrolidin-1-ylmethyl-ethyl]-amide-L-tartaric acid salt] is a proprietary compound supplied by Genzyme Corporation.

Male Sprague-Dawley rats were obtained from the animal facility at the School of Veterinarian, Universidad de Buenos Aires. The rats were individually housed under controlled conditions of illumination, humidity, and temperature, with food and water being available ad libitum. Animals were allowed 3-7 d to adapt to housing conditions before undergoing any manipulation.

Stx 2 supernatant. The stx2 gene was cloned into pGEM-T-Easy, and recombinant E. coli $\mathrm{DH} 5 \alpha$ expressing Stx 2 were cultured overnight at $37^{\circ} \mathrm{C}$ in Luria-Bertani broth supplemented with $100 \mu \mathrm{g} / \mathrm{mL}$ ampicillin (Sigma Chemical Co.-Aldrich Co., St. Louis, MO). Bacterial cells were then removed by centrifugation, and the supernatant from recombinant $E$. coli expressing Stx2 (sStx2) was filtered through $0.22 \mu \mathrm{m}$ pore size filter units (Millipore Corp., Billerica, MA). A control supernatant (sCtrl) was prepared from a recombinant $E$. coli that contained only the plasmid.

Cytotoxic activity of sStx2 was measured by neutral red uptake in Vero cells treated with different dilutions of sStx 2 for $72 \mathrm{~h}$ (12) and the lipopolysaccharide (LPS) content was determined using the HEK-Blue LPS Detection Kit (InvivoGen, San Diego, CA). Sample supernatants contained 75 ng $\mathrm{LPS} / \mu \mathrm{g}$ of Stx2 protein.

The sStx2 was also run on a $12.5 \%$ SDS polyacrylamide gel and electrotransferred to a nitrocellulose membrane. Immunoblot analysis using the MAb against the A and B subunits (Biodesign International, Saco, ME) of Stx2, showed two bands of $32 \mathrm{kD}$ and $7.7 \mathrm{kD}$ that correspond to Stx2A and Stx2B, respectively (20).

Experimental model of HUS in rats. Animal studies were conducted using protocols approved by the Institutional Animal Care and Use Committee and Scientific Program of the Universidad de Buenos Aires. Rats were allowed to acclimate for 3-7 d in metabolic cages with water and food ad libitum before treatments. For C-9 therapy, C-9 was dissolved in water and added to standard rat chow to give a concentration of $1 \mathrm{mg} \mathrm{C}-9 / \mathrm{g}$ of dry food.

Rats were randomly divided in four groups of recently weaned male Sprague-Dawley rats (70-100 g, 4 wk old). To develop a model of HUS in rats, animals were injected i.p. with $1 \mathrm{~mL} / 100 \mathrm{~g}$ body weight of sStx2 (400 ng Stx $2 / \mathrm{mL}$ and $30 \mathrm{ng} \mathrm{LPS} / \mathrm{mL}$ ). Some of these rats were orally treated with C-9 during $6 \mathrm{~d}$, starting $2 \mathrm{~d}$ prior until $4 \mathrm{~d}$ after sStx 2 injection. Other animals were orally treated with C-9 and injected with sCtrl. Control rats were injected with sCtrl and did not receive C-9 treatment. Survival analysis was performed in all groups of rats. Water drinking, food intake, and body weight were daily monitored since $2 \mathrm{~d}$ prior until $14 \mathrm{~d}$ after the i.p. injection.

For morphological studies, rats were i.p. anesthetized (100 $\mu \mathrm{g}$ ketamine and $10 \mu \mathrm{g}$ diazepam/g body weight) $2 \mathrm{~d}$ after sStx 2 injection. Then, they were perfused with $10 \%$ formol in PBS $0.1 \mathrm{M}(\mathrm{pH}$ 7.4) followed by the removal of kidneys and colon. Urine and blood samples were obtained before killing the animals. Urea and creatinine concentrations in serum were measured with commercial kits (Wierner Laboratory, Argentina).

Histopathology analysis. The kidney and colon were quickly extracted and fixed in $10 \%$ formol in PBS $0.1 \mathrm{M}$ ( $\mathrm{pH} 7.4)$. The tissue sections were dehydrated and embedded in paraffin. Sections of $5 \mu \mathrm{m}$ were made with a microtome (Leica RM 2125, Wetzlar, Germany) and mounted on 2\% silanecoated slides. Sections stained with hematoxylin-eosin, periodic acid-Schiff, Jones methenamine silver, and phosphotungstic acid hematoxylin were observed by light microscopy (Nikon Eclipse 200, Melville, NY).

Quantitation of Gb3 and GL1 in kidney. Rats were randomly separated in different groups; some were orally treated with C-9 (1 mg C-9/g of dry food, 10-15 mg C-9/rat/d) from 1 to $4 \mathrm{~d}$, whereas others were not. Kidneys were removed from anesthetized rats, immediately frozen, and kept at $-70^{\circ} \mathrm{C}$ until GL1 and Gb3 quantitations were performed. Rat kidney tissues were homogenized in iced water using a bead-beater homogenizer (BioSpec Products, Inc., Bartesville, OK). Sphingolipids were extracted by mixing the homogenate with 50 -fold of acetonitrile/methanol $(9: 1 \mathrm{vol} / \mathrm{vol})$. The mixture was vortexed, sonicated, and centrifuged at $8300 \mathrm{~g}$ for $3 \mathrm{~min}$ at room temperature. The supernatant was transferred to HPLC vials for analysis. GL1 and Gb3 were quantified by a UPLC (Waters Corp. Medford, MA) hyphenated to an API-4000 mass spectrometer (MDS Applied BioSystems, Framingham, MA) running in multiple reaction monitoring (MRM) mode. GL1 and Gb3 standards were purchased from Matreya, Inc (Pleasant Gap, PA). To separate GL1 from GalCer, Atlantis HILIC silica column $(3 \mu \mathrm{m}, 150 \times 3.0 \mathrm{~mm}$, Waters Corp.) was used. For Gb3 analysis, Aquity HILIC column $(1.7 \mu \mathrm{m}, 100 \times 3.0$ $\mathrm{mm}$, Waters Corp.) was used. Mobile phase A consisted of 97:1:1:1 acetonitrile/methanol/water/acetic acid and $5 \mathrm{mM}$ ammonium acetate. Mobile phase B consisted of 98:1:1 methanol/water/acetic acid with $5 \mathrm{mM}$ ammonium acetate). The mass spectrometry detection was performed in positive ion mode. For GL1 analysis, the seven most abundant isoforms were monitored using the following MRM transitions: $\mathrm{m} / \mathrm{z} 700.6>\mathrm{m} / \mathrm{z} 264.3 ; \mathrm{m} / \mathrm{z} 728.6>$ $\mathrm{m} / \mathrm{z} 264.3 ; \mathrm{m} / \mathrm{z} 756.7>\mathrm{m} / \mathrm{z} 264.3 ; \mathrm{m} / \mathrm{z} 784.7>\mathrm{m} / \mathrm{z} 264.3 ; \mathrm{m} / \mathrm{z} 798.7>\mathrm{m} / \mathrm{z}$ 264.3; m/z $810.8>\mathrm{m} / \mathrm{z} 264.3$; and $\mathrm{m} / \mathrm{z} 812.8>\mathrm{m} / \mathrm{z} 264.3$. For Gb3 analysis, 12 MRM transitions were monitored: $\mathrm{m} / \mathrm{z} 1024.70>\mathrm{m} / \mathrm{z} 264.3 ; \mathrm{m} / \mathrm{z}$ $1052.70>\mathrm{m} / \mathrm{z} 264.3 ; \mathrm{m} / \mathrm{z} 1080.70>\mathrm{m} / \mathrm{z} 264.3 ; \mathrm{m} / \mathrm{z} 1106.70>264.3 ; \mathrm{m} / \mathrm{z}$ $1,108.70>\mathrm{m} / \mathrm{z} 264.3 ; \mathrm{m} / \mathrm{z} 1122.70>\mathrm{m} / \mathrm{z} 264.3 ; \mathrm{m} / \mathrm{z} 1124.70>\mathrm{m} / \mathrm{z} 264.3$ $\mathrm{m} / \mathrm{z} 1132.70>\mathrm{m} / \mathrm{z} 264.3 ; \mathrm{m} / \mathrm{z} 1134.70>\mathrm{m} / \mathrm{z} 264.3 ; \mathrm{m} / \mathrm{z} 1136.70>\mathrm{m} / \mathrm{z}$ 264.3; and m/z $1150.70>\mathrm{m} / \mathrm{z} 264.3 \mathrm{~m} / \mathrm{z} 1152.70>\mathrm{m} / \mathrm{z} 264.3$. The area counts for each isoform were summed to obtain the total GL-1 or Gb3 area counts. All data were normalized to the control.

\section{RESULTS}

Quantitation of GL1and Gb3 in kidney. A significant inhibition of approximately 58\% in GL1 expression levels was observed in rat kidneys after $1 \mathrm{~d}$ of C-9 treatment compared with nontreated rats $(3.9 \pm 0.1$ versus $9.3 \pm 1.2 \mu \mathrm{g} / \mathrm{g}$ kidney, respectively; Fig. 1A). In the same kidney samples, an inhibition of $\sim 30 \%$ was also observed in $\mathrm{Gb} 3$ expression levels (Fig. $1 B$ ). The renal GL1 and Gb3 levels remained inhibited after 2 and $4 \mathrm{~d}$ of C-9 treatment, showing that there is a trend for greater decrease in Gb3 levels with more days of C-9 treatment (Fig. 1B).

Survival analysis. Rats i.p. injected with sStx 2 died 3-5 d after injection (Fig. 2). The oral treatment with C-9 administered during $6 \mathrm{~d}$, from $2 \mathrm{~d}$ prior until $4 \mathrm{~d}$ after sStx 2 i.p. injection, prevented the mortality of $50 \%$ of the rats. The remaining $50 \%$ of rats treated with C-9 died 3-6 d after sStx2 injection (Fig. 2). All rats injected with $\mathrm{sCtrl}$, whether treated with C-9 or not, survived (Fig. 2). Beginning the treatment with C-9 at the same time or even $1 \mathrm{~d}$ before Stx 2 injection did not prevent the mortality of the rats (data not shown).

Curves in Figure 3 represent the time course of water drinking, food intake, and body weight obtained from rats i.p.
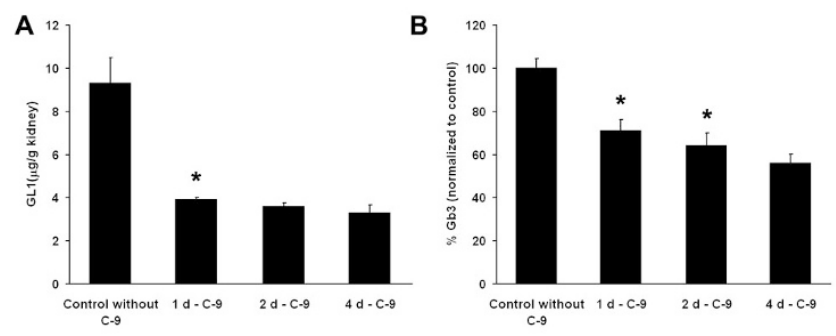

Figure 1. Effects of oral treatment with C-9 on GL1 (A) and Gb3 (B) expression levels in rat kidneys. Each bar represents the mean $\pm \mathrm{SEM}, n=$ 4 rats. $t$ test indicates significant differences $(* p<0.05 ; * * p<0.01)$ for rat kidneys treated at different times with C-9 compared with control animals without C-9 treatment. 
injected with sStx2 or sCtrl, and orally treated or not with C-9. The animals injected with sStx2 immediately decreased water and food intake, although those treated with C-9 recovered water and food intake 3-4 d later (Fig. 3A and $B$ ). The C-9 treatment in rats injected with sCtrl did not change water and food intake along the days compared with rats that did not received C-9 treatment. Rats treated and nontreated with C-9 lost about $11 \%$ of body weight $2 \mathrm{~d}$ after sStx 2 injection but those treated with the drug started to gain weight about $6 \mathrm{~d}$ after sStx2 inoculation (Fig. 3C).

The serum $\mathrm{Na}^{+}$levels in sStx2 injected rats with and without C-9 treatment (146 \pm 9 and $128 \pm 7 \mathrm{mEq} / \mathrm{L}$, respectively) were not significantly different from the $\mathrm{Na}^{+}$values found in $\mathrm{sCtrl}$ injected rats $(136 \pm 6 \mathrm{mEq} / \mathrm{L})$, indicating that the water balance was not modified. Urine excretion significantly decrease in sStx2-injected rats compared with sCtrl $(6.1 \pm 1$ versus $12.9 \pm 2.3 \mathrm{~mL} / \mathrm{d}, n=6, p<0.05)$ but was not modified in those rats treated with sStx 2 and C-9 (10.5 \pm $4.4 \mathrm{~mL} / \mathrm{d})$.

Creatinine and urea in serum. As shown in Figure $4 A$, there was a significant rise in serum creatinine levels of rats $2 \mathrm{~d}$ after sStx2 injection, compared with sCtrl injected rats $(10.83 \pm 1.65 \mathrm{mg} / \mathrm{L}$ versus $4.181 \pm 1.04 \mathrm{mg} / \mathrm{L}, n=6, p<$ 0.05). A significant increase in serum urea levels was also observed in the same group of rats $2 \mathrm{~d}$ after sStx 2 injection,

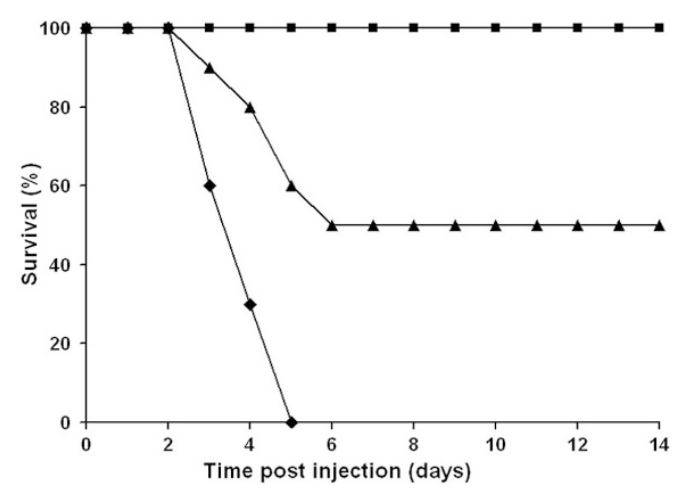

Figure 2. Survival curves of rats after i.p. injected with sStx2, orally treated $(\boldsymbol{\Delta})$ or nontreated $(\bullet)$ with C-9. The mortality of rats treated with C-9 from $2 \mathrm{~d}$ before $4 \mathrm{~d}$ after sStx 2 injection was $50 \%$. The remaining $50 \%$ of rats treated with C-9 died between 3 and $6 \mathrm{~d}$ after sStx 2 injection. A $100 \%$ of survival was observed in rats injected with sCtrl and treated with C-9 (ם). compared with sCtrl injected rats $(2.83 \pm 0.58 \mathrm{~g} / \mathrm{L}$ versus $0.35 \pm 0.06 \mathrm{~g} / \mathrm{L}, n=6, p<0.05$, in Fig. $4 B$ ). Both results indicated the development of renal failure produced by sStx 2 . The oral treatment with C-9 prevented the increase of both creatinine and urea levels in serum of rats injected with sStx2 (Fig. $4 A$ and $B$ ), showing the protective effect of C-9. Both serum creatinine and urea levels in rats treated with C-9 and injected with sCtrl remained similar to those obtained in animals that did not receive the C-9 (Fig. $4 A$ and $B$ ).

Histological observations. Tubular necrosis and glomerular mesangial proliferation were observed in rat kidney, $2 \mathrm{~d}$ after sStx 2 injection (Fig. $5 B$ and $C$ ). The C-9 treatment decreased the tubular necrosis produced by sStx2, showing a significantly less extension of the renal damage (Fig. 5E). Normal glomeruli and tubules were observed in the rats injected with sCtrl treated or not treated with C-9 (Fig. 5A and $D$, respectively). Capillary wall thickening with double basal membranes, and fibrin within capillary lumen observed in some isolated glomeruli of sStx2-injected rats (Fig. $6 A$ and $C$, respectively) were not present in Stx2-injected rats treated with C-9 (Fig. $6 B$ and $D$ ).

In colon, sStx 2 produced a focal decrease of goblet cells in the upper two thirds of the crypts (Fig. 7C). The treatment with C-9 prevented the alteration in the colonic goblet cells (Fig. 7D). The colon superficial epithelium was conserved in the four groups of rats. Normal distribution of goblet cells was observed in rats injected with $\mathrm{sCtrl}$, treated or not treated with
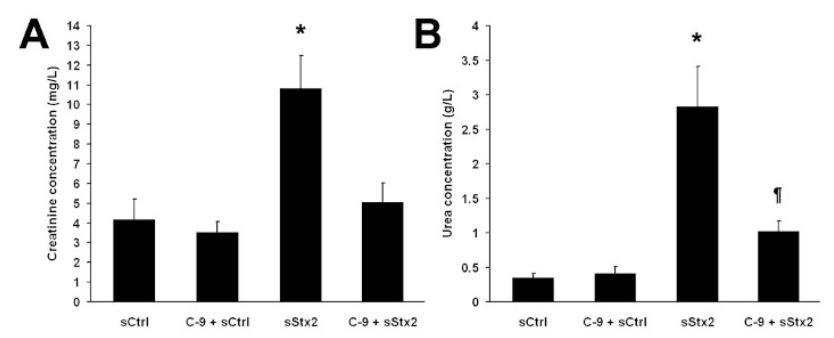

Figure 4. (A) Serum creatinine concentration and $(B)$ serum urea concentration. The significant increase in serum creatinine and urea concentrations in rats injected with sStx 2 was prevented by the oral treatment with $\mathrm{C}-9$ since $2 \mathrm{~d}$ before $\mathrm{sStx} 2$ injection. Each bar represents the mean $\pm \mathrm{SEM}, n=6$ rats. $t$ test indicates significant differences for rats injected with sStx 2 compared with the other groups $(* p<0.05)$ and for C-9 + sStx2 compared with sCtrl ( $I p<0.05)$.
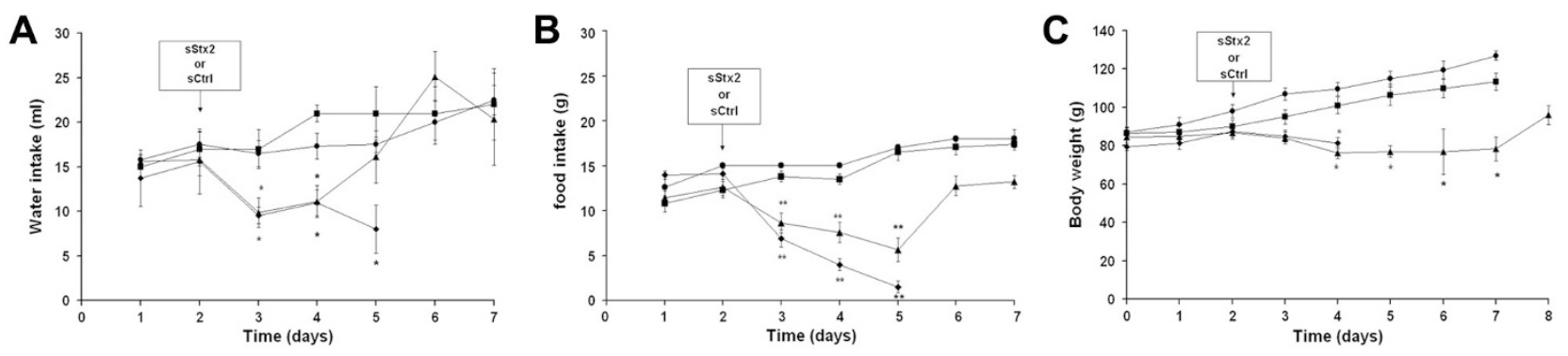

Figure 3. Time course of water intake $(A)$, food intake $(B)$, and body weight $(C)$ from rats i.p. injected with sStx 2 or sCtrl, and orally treated or not with C-9. For C-9 therapy, C-9 was added to standard rat chow to give a concentration of $1 \mathrm{mg} \mathrm{C}-9 / \mathrm{g}$ of dry food. Each point of the curves represents the mean \pm SEM, $n=10$. The unpaired $t$ test was used to calculate differences at each time point between experimental groups. In $(A) * p<0.05$ for sStx2 $(\checkmark)$ and C-9 $+\mathrm{sStx} 2$

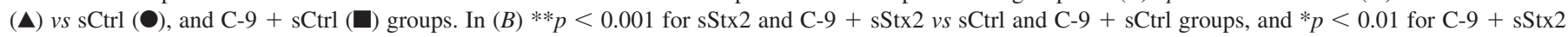
vs sCtrl and C-9 + sCtrl groups. In $(C) * p<0.01$ for sStx2 and C-9 + sStx2 vs sCtrl and C-9 + sCtrl groups. 


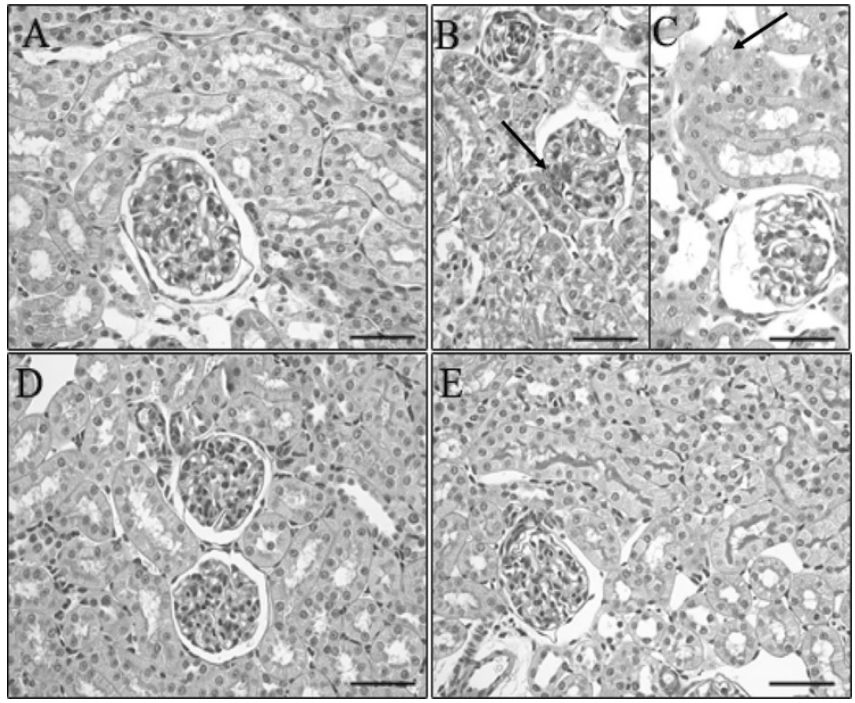

Figure 5. Histological observation of rat kidney sections. Glomerular mesangial proliferation (black arrow in $B$ ) and tubular necrosis (black arrow in $C$ ) were observed in kidneys from rats injected with sStx2. The C-9 treatment showed a significantly less extension of renal damage $(D)$. Normal glomeruli and renal tubules were observed in $\mathrm{sCtrl}(A)$ and in $\mathrm{C}-9+\mathrm{sCtrl}(E)$ groups. [periodic acid-Schiff (PAS); scale bar $=50 \mu \mathrm{m}$ ].
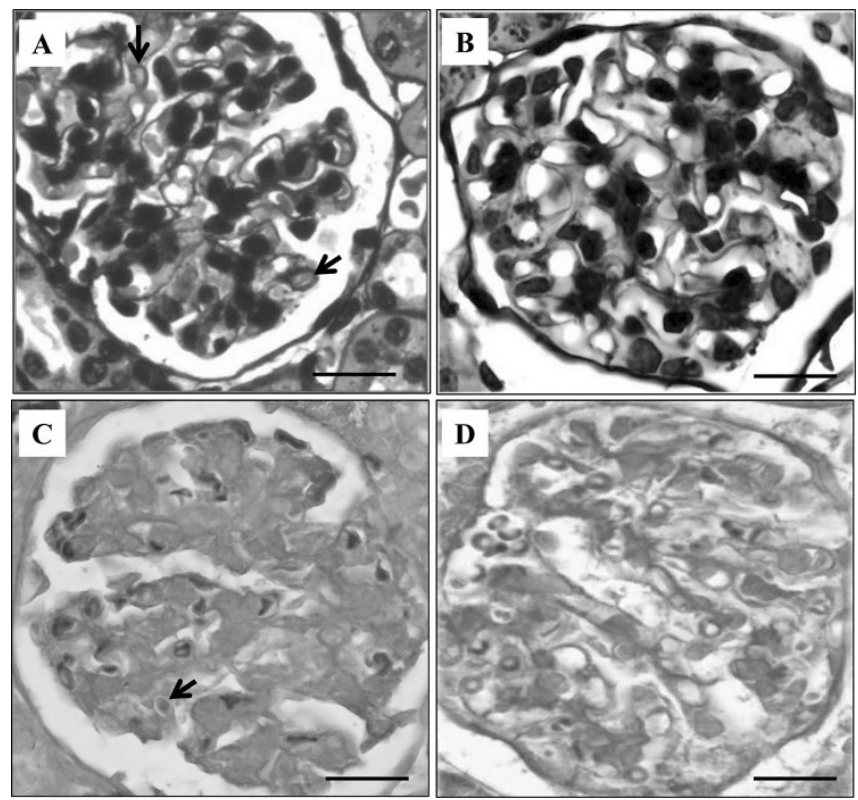

Figure 6. Capillary wall thickening with double basal membranes (black arrows in $A$; Jones methenamine silver) and fibrin within capillary lumen (black arrow in $C$; phosphotungstic acid hematoxylin) were observed in Stx2-treated rats. These lesions were not observed in rats treated with C-9 and sStx2 $(B$ and $D)$. Scale bar $=20 \mu \mathrm{m}$.

C-9 (Fig. 7A and $B$, respectively). The watery diarrhea observed in rats injected with $\mathrm{sStx} 2$ was not found when the rats were treated with C-9.

\section{DISCUSSION}

Although many laboratories have made efforts to develop an effective treatment for Stx-mediated HUS (21), a specific therapy has not been found yet. Compounds mimicking the Gb3 receptor (22-25) and antibodies against Stx subunits

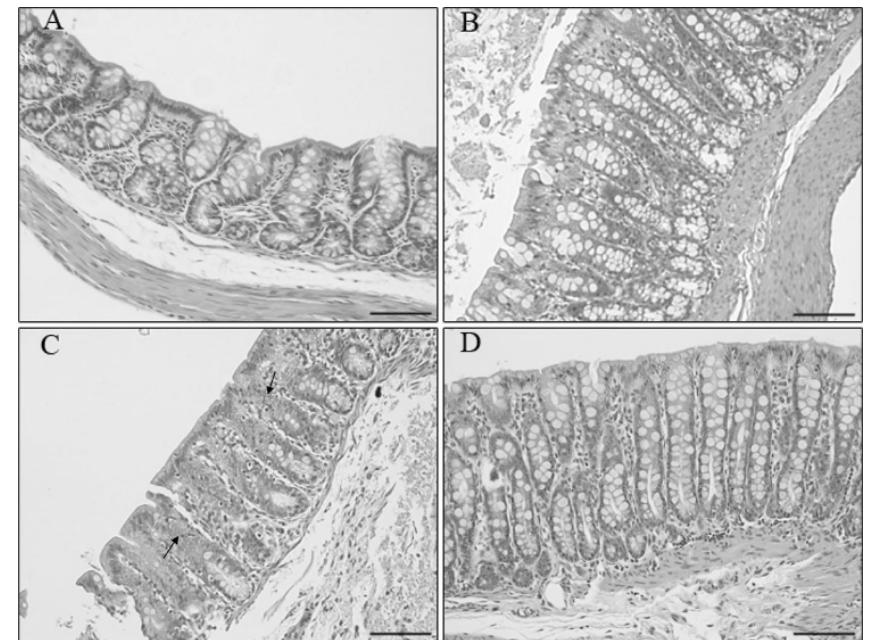

Figure 7. Histological observation of rat colon sections obtained from the following groups. Stx2 produced a decrease of goblet cells in colonic epithelium (black arrow in $C$ ). The treatment with C-9 prevented the effects of Stx2 in colon $(D)$, showing a normal distribution of goblet cells, as seen in $\mathrm{sCtrl}(A)$ and $\mathrm{C}-9+\mathrm{sCtrl}(B)$ groups. The colon epithelium was conserved in the four groups of rats (hematoxylin-eosin; scale bar $=100 \mu \mathrm{m}$ ).

(26-28) are some of the different neutralizing agents that have been assayed to prevent Stx 1 and/or Stx2 effects in humans or experimental animal models of HUS.

In this study, we have demonstrated that oral treatment with C-9, a potent inhibitor of GL1 synthase and Gb3, decreased rat mortality to $50 \%$ and reduced renal damage and intestinal alterations caused by the toxin. The C-9 was effective when given during $6 \mathrm{~d}$, starting $2 \mathrm{~d}$ prior until $4 \mathrm{~d}$ after sStx 2 i.p. injection. Renal damage with tubular necrosis, glomerular mesangial proliferation, and a concomitant significantly increase of creatinine and urea in serum, and oliguria were detected in weaned rats injected with sStx2. These physiological parameters and renal damages, that were comparable to those previously described (29), were partially prevented with C-9. We have demonstrated that Stx2 affects not only the kidney but also the brain of the rats (20) where an increase of $\mathrm{Gb} 3$ expression was observed (30). Rats have probably died as a result of both, kidney and extra kidney susceptibility to Stx2. The fact that C-9 prevents only $50 \%$ of rat mortality may be due to the inability of this compound to cross the blood brain barrier, so the rats could have died from neurological injuries. However, other causes observed in patients with HUS such as hyperkalemia, pulmonary edema, hypertension, and cardiac failure cannot be discarded (1).

Although sStx2-treated weaned rats developed glomerular mesangial proliferation, the glomerular thrombotic microangiopathic lesions, characteristic of HUS were scarcely observed. A significant tubular injury without glomerular thrombotic microangiopathic lesions was described in Stx2-treated rodents possibly because $\mathrm{Gb} 3$ was not detected in the glomerular endothelial cells (31). However, a low expression of Gb3 cannot be discarded unless more sensitive techniques are used (32).

The protective effects against sStx 2 were observed when C-9 was given daily, dissolved into the rat chows. Before sStx2 injection, rats ate about $10-15 \mathrm{~g}$ chow/rat/d containing 10-15 mg C-9/rat/d. After sStx2 injection, rats lost about $11 \%$ 
of body weight mainly because they decreased food intake. The food intake dropped to about $5 \mathrm{~g}$ chow/rat/d indicating a proportional decrease in C-9 intake. Despite the fact that C-9 intake decreased to $5 \mathrm{mg} / \mathrm{rat} / \mathrm{d}$, this dose was enough to protect the rats from Stx 2 cytotoxic effects and to recover their weight 6 d later. When C-9 was given in the water instead of in the food, the compound protected the rats from Stx2 damage in intestinal and renal tissues but did not prevent their mortality [Silberstein C. et al., Effects of a GL1 synthase inhibitor on an experimental model of HUS in rats, 2009 VTEC2009, 7th International Symposium on Shiga toxin (Verocytotoxin)producing Escherichia coli infection, May 10-13, 2009, Buenos Aires, Argentina, Abstract P04.3.4]. The results in vivo are consistent with those previously reported in vitro in primary cultures of HRTEC (14). Preincubation of HRTEC for $2 \mathrm{~d}$ with C-9 prevented the cytotoxic effect of Stx2. Under this condition, a significant inhibition in the levels of Gb3 was observed (14). In this study, an inhibition of GL1 and Gb3 levels was also observed in rat kidneys 1-4 d after oral treatment with $\mathrm{C}-9$. We have also seen that $\mathrm{C}-9$ prevented the focal loss of goblet cells observed in colon of Stx 2 injected rats. It was reported that an inhibitor of intracellular glycolipid biosynthesis decreased goblet cell loss in colon of mice with inflammatory bowel disease, causing antiinflammatory activity (33). In this work, the treatment with C-9 could protect the colonic mucosa against inflammatory responses triggered by sStx 2 by decreasing the expression levels of Gb3 in the leukocytes and, in consequence, the inflammation associated to these cells $(34,35)$.

In summary, we here propose that prevention of $\mathrm{Gb3}$ synthesis by GL1 synthase inhibitors such as C-9 could be a novel substrate inhibition therapy to prevent Stx 2 action in target cells. Considering that there is a $3-5 \mathrm{~d}$ period of time between the initial gastrointestinal symptoms and the development of HUS, a treatment with C-9 for a few days within that period of time would be a possible therapeutic strategy to prevent the renal failure observed in children with HUS.

Acknowledgment. We thank Dr. Mohamed Karmali (Laboratory for Foodborne Zoonoses, Public Health Agency of Canada, ON, Canada) for suggestions and advice.

\section{REFERENCES}

1. Repetto HA 1997 Epidemic hemolytic-uremic syndrome in children. Kidney Int 52:1708-1719

2. Repetto HA 2005 Long-term course and mechanisms of progression of renal disease in hemolytic uremic syndrome. Kidney Int Suppl (97):S102-S106

3. Gianantonio C, Vitacco M, Mendiaharzu F, Rutty A 1964 The hemolytic-uremic syndrome. J Pediatr 64:478-491

4. Rivas M, Miliwebsky E, Chinen I, Deza N, Leotta G 2006 [The epidemiology of hemolytic uremic syndrome in Argentina. Diagnosis of the etiologic agent, reservoirs and routes of transmission]. Medicina (B Aires) 66:27-32

5. Lingwood CA 1996 Role of verotoxin receptors in pathogenesis. Trends Microbiol 4:147-153

6. Rutjes NW, Binnington BA, Smith CR, Maloney MD, Lingwood CA 2002 Differential tissue targeting and pathogenesis of verotoxins 1 and 2 in the mouse animal model. Kidney Int 62:832-845

7. Okuda T, Tokuda N, Numata S, Ito M, Ohta M, Kawamura K, Wiels J, Urano T, Tajima O, Furukawa K, Furukawa K 2006 Targeted disruption of Gb3/CD77 synthase gene resulted in the complete deletion of globo-series glycosphingolipids and loss of sensitivity to verotoxins. J Biol Chem 281:10230-10235

8. Karpman D, Hakansson A, Perez MT, Isaksson C, Carlemalm E, Caprioli A, Svanborg C 1998 Apoptosis of renal cortical cells in the hemolitic-uremic syndrome: in vivo and in vitro studies. Infect Immun 66:636-644
9. Creydt VP, Silberstein C, Zotta E, Ibarra C 2006 Cytotoxic effect of Shiga toxin-2 holotoxin and its B subunit on human renal tubular epithelial cells. Microbes Infect 8:410-419

10. Uchida H, Kiyokawa N, Horie H, Fujimoto T, Takeda T 1999 The detection of Shiga toxins in the kidney of patients with hemolytic uremic syndrome. Pediatr Res 45:133-137

11. Kaneko K, Kiyokawa N, Ohtomo Y, Nagaoka R, Yamashiro Y, Taguchi T, Mori T, Fujimoto J, Takeda T 2001 Apoptosis of renal tubular cells in Shiga-toxin mediated hemolytic uremic syndrome. Nephron 87:182-185

12. Silberstein C, Pistone Creydt V, Gerhard E, Núñez P, Ibarra C 2008 Inhibition of water absorption in human proximal tubular epithelial cells in response to Shiga toxin-2. Pediatr Nephrol 23:1981-1990

13. Creydt VP, Miyakawa MF, Martín F, Zotta E, Silberstein C, Ibarra C 2004 The Shiga toxin 2 B subunit inhibits net fluid absorption in human colon and elicits fluid accumulation in rat colon loops. Braz J Med Biol Res 37:799-808

14. Silberstein C, Copeland DP, Chiang WL, Repetto HA, Ibarra C 2008 A glucosylceramide synthase inhibitor prevents the cytotoxic effects of shiga toxin- 2 on human renal tubular epithelial cells. J Epithelial Biol Pharmacol 1:71-75

15. Lee L, Abe A, Shayman JA 1999 Improved inhibitors of glucosylceramide synthase. J Biol Chem 274:14662-14669

16. Kolter T, Proia RL, Sandhoff K 2002 Combinatorial ganglioside biosynthesis. J Biol Chem 277:25859-25862

17. Abe A, Gregory S, Lee L, Killen PD, Brady RO, Kulkarni A, Shayman JA 2000 Reduction of globotriaosylceramide in Fabry disease mice by substrate deprivation. J Clin Invest 105:1563-1571

18. McEachern KA, Fung J, Komarnitsky S, Siegel CS, Chuang W-L, Hutto E, Shayman JA, Grabowski GA, Aerts JM, Cheng SH, Copeland DP, Marshall JA 2007 A specific and potent inhibitor of glucosylceramide synthase for substrate inhibition therapy of Gaucher disease. Mol Genet Metab 91:259-267

19. Zhao H, Przybyska M, Wu I-H, Zhang J, Siegel C, Komarnitsky S, Yew NS, Cheng SH 2007 Inhibiting glycosphingolipid synthesis improves glycemic control and insulin sensitivity in animal model of type 2 diabetes. Diabetes 56:1210-1218

20. Goldstein J, Loid CF, Pistone Creydt V, Boccoli J, Ibarra C 2007 Intracerebroventricular administration of Shiga toxin type 2 induces striatal neuronal death and glia alterations: an ultrastructural study. Brain Res 1161:106-115

21. Rogers TJ, Paton JC 2009 Therapeutic strategies for Shiga toxin-producing Escherichia coli infections. Expert Rev Anti Infect Ther 7:683-686

22. Yamagami S, Motoki M, Kimura T, Izumi H, Takeda T, Katsuura Y, Matsumoto Y 2001 Efficacy of postinfection treatment with anti-Shiga toxin (Stx) 2 humanized monoclonal antibody TMA-15 in mice lethally challenged with Stx-producing Escherichia coli. J Infect Dis 184:738-742

23. Paton JC, Rogers TJ, Morona R, Paton AW 2001 Oral administration of formaldehyde-killed recombinant bacteria expressing a mimic of the Shiga toxin receptor protects mice from fatal challenge with Shiga-toxigenic Escherichia coli. Infect Immun 69:1389-1393

24. Trachtman H, Cnaan A, Christen E, Gibbs K, Zhao S, Acheson DW, Weiss R, Kaskel FJ, Spitzer A, Hirschman GH 2003 Effect of an oral Shiga toxin-binding agent on diarrhea-associated hemolytic uremic syndrome in children: a randomized controlled trial. JAMA 290:1337-1344

25. Neri P, Tokoro S, Yokoyama S-I, Miura T, Murata T, Nishida Y, Kajimoto T, Tsujino S, Inazu T, Usui T, Mori H 2007 Monovalent Gb3-/Gb2_-derivatives conjugated with a phosphatidyl residue: a novel class of Shiga toxin-neutralizing agent. Biol Pharm Bull 30:1697-1701

26. Ishikawa S, Kawahara K, Kagami Y, Isshiki Y, Kaneko A, Matsui H, Okada N, Danbara H 2003 Protection against Shiga toxin 1 challenge by immunization of mice with purified mutant Shiga toxin 1. Infect Immun 71:3235-3239

27. Capozzo AV, Pistone Creydt V, Dran G, Fernández G, Gomez S, Bentancor LV, Rubel C, Ibarra C, Isturiz M, Palermo MS 2003 Development of genetic vaccines against hemolytic uremic syndrome (HUS). Infect Immun 71:3971-3978

28. Tsuji T, Shimizub T, Sasakia K, Tsukamotoa K, Arimitsua H, Ochia S, Shimizua T, Taniguchic K, Nodab M, Nerid P, Morid H 2008 A nasal vaccine comprising B-subunit derivative of Shiga toxin 2 for cross-protection against Shiga toxin types 1 and 2. Vaccine 26:2092-2099

29. Zotta E, Lago N, Ochoa F, Repetto HA, Ibarra C 2008 Development of an experimental hemolytic uremic syndrome in rats. Pediatr Nephrol 23:559-567

30. Tironi-Farinati C, Loidl CF, Boccoli J, Parma Y, Fernandez Miyakawa ME, Goldstein J 2010 Intracerebroventricular Shiga toxin 2 increases the expression of its receptor globotriaosylceramide and causes dendritic abnormalities. J Neuroimmunol 222:48-61

31. Ray PE, Liu X-H 2001 Pathogenesis of Shiga toxin-induced hemolytic uremic syndrome. Pediatr Nephrol 16:823-839

32. Zumbrun SD, Hanson L, Sinclair JF, Freedy J, Melton-Celsa AR, RodriguezCanales J, Hanson JC, O'Brien AD 2010 Human intestinal tissue and cultured colonic cells contain globotriaosylceramide synthase mRNA and the alternate Shiga toxin receptor, globotetraosylceramide. Infect Immun 78:4488-4499

33. Shen C, Bullens D, Kasran A, Maerten P, Boon L, Aerts JM, van Assche G, Geboes $\mathrm{K}$, Rutgeerts P, Ceuppens JL 2004 Inhibition of glycolipid biosynthesis by $\mathrm{N}$-(5adamantane-1-yl-methoxy-pentyl)-deoxynojirimycin protects against the inflammatory response in hapten-induced colitis. Int Immunopharmacol 4:939-951

34. van de Kar NC, Monnens LA, Karmali MA, van Hinsbergh VW 1992 Tumor necrosis factor and interleukin-1 induce expression of the verocytotoxin receptor globotriaosylceramide on human endothelial cells: implications for the pathogenesis of the hemolytic uremic syndrome. Blood 80:2755-2764

35. Schüller S, Frankel G, Phillips AD 2004 Interaction of Shiga toxin from Escherichia coli with human intestinal epithelial cell lines and explants: Stx2 induces epithelial damage in organ culture. Cell Microbiol 6:289-301 